\title{
Peripheral circulation miRNA expression of pediatric brain tumors and its relation to tumor miRNA expression levels
}

\author{
Markus Bookland, MD, ${ }^{1,2}$ Eileen Gillan, MD, ${ }^{3}$ Xianyuan Song, MD, ${ }^{4}$ and Antonina Kolmakova, $\mathrm{PhD}^{2}$ \\ Divisions of ${ }^{1}$ Neurosurgery and ${ }^{3}$ Hematology-Oncology, Connecticut Children's, Hartford; ${ }^{2}$ Department of Pediatrics, University of \\ Connecticut Health Center, Farmington; and 4Department of Pathology \& Lab Medicine, Hartford Hospital, Hartford, Connecticut
}

\begin{abstract}
OBJECTIVE Micro RNAs (miRNAs) in peripheral biofluids (e.g., blood, saliva, urine) have been investigated as potential sources of diagnostic and prognostic information for a variety of tumor types, including pediatric brain tumors. While significant predictive associations have been identified between unique serum miRNA concentrations and some pediatric brain tumors, it is unclear whether serum miRNA abnormalities in pediatric brain tumor patients are representative of miRNA alterations in the tumor tissue compartment or whether they represent host tissue reactions to the presence of a brain tumor. The authors sought to identify whether serum miRNA changes in pediatric brain tumor patient sera could be explained by miRNA alterations within their tumors.
\end{abstract}

METHODS Matched serum and tissue samples were taken from a cohort of pediatric brain tumor patients (juvenile pilocytic astrocytoma $[\mathrm{JPA}]=3$, medulloblastoma $=4$, ependymoma $=3$ ), and unmatched control samples $(n=5)$ were acquired from control pediatric patients without oncological diagnoses. Extracted RNAs were tested within an array of 84 miRNAs previously noted to be relevant in a variety of brain tumors.

RESULTS miR-26a-5p correlated strongly in JPA patients within both the serum and tumor tissue samples $\left(R^{2}=0.951\right.$, $p=0.046$ ), and serum levels were highly predictive of JPA (area under the curve $=0.751, p=0.027$ ). No other miRNAs that were significantly correlated between biological compartments were significantly associated with brain tumor type. In total, 15 of 84 tested miRNAs in JPA patients, 14 of 84 tested miRNAs in ependymoma patients, and 4 of 84 tested miRNAs in medulloblastoma patients were significantly, positively correlated between serum and tumor tissue compartments $\left(R^{2}>0.950, p<0.05\right)$.

CONCLUSIONS The majority of miRNA changes in pediatric brain tumor patient sera that are significantly associated with the presence of a brain tumor do not correlate with brain tumor miRNA expression levels. This suggests that peripheral miRNA changes within pediatric brain tumor patients likely derive from tissues other than the tumors themselves.

https://thejns.org/doi/abs/10.3171/2020.2.PEDS19715

KEYWORDS biomarker; medulloblastoma; miRNA; serum; tissue; ependymoma; juvenile pilocytic astrocytoma; oncology

$\mathrm{T}$ HE use of micro RNAs (miRNAs) in various biofluids to predict the presence and potential malignancy of cancers has been demonstrated in a wide variety of adult and pediatric tumors. ${ }^{1-8}$ In the case of several adult tumors, including hepatocellular carcinoma, ${ }^{9}$ prostate cancer ${ }^{10}$ and squamous cell cancer, ${ }^{11}$ there is evidence that serum miRNA expression levels also strongly correlate with miRNA expression within matched tumor tissues. However, the association between the serum and tissue miRNomes of brain tumor patients is not understood. As a re- sult, conclusions about whether changes in serum miRNA represent changes in the tumor biology or changes in the host biology cannot be drawn with any certainty. Without this understanding, neurosurgeons and neurooncologists cannot be certain that aberrations in serum miRNA expression levels truly represent the presence of oncological tissues, and they cannot discern whether miRNA changes during therapy are the result of treatment impact on the tumor or the patient. In short, understanding the derivation of serum miRNA changes in pediatric brain tumor patients

ABBREVIATIONS AUC = area under the curve; JPA = juvenile pilocytic astrocytoma; miRNA = micro RNA; PCR = polymerase chain reaction; $R O C=$ receiver operating characteristic; $\mathrm{RT}=$ real time.

SUBMITTED December 4, 2019. ACCEPTED February 27, 2020.

INCLUDE WHEN CITING Published online May 8, 2020; DOI: 10.3171/2020.2.PEDS19715. 
does not preclude their development for biomarker purposes, but it is essential for their appropriate application. This holds particular significance in the case of pediatric and adult brain tumors where molecular subtyping of gliomas and medulloblastomas can have a profound impact on prognosis and therapeutic management, ${ }^{12,13}$ raising the diagnostic bar for any liquid biopsy technology meant to address these tumors, including miRNA.

It is not clear that miRNAs circulating in the sera of pediatric brain tumor patients can be expected to accurately represent miRNA expression patterns in the tumor tissues themselves, as the blood-brain barrier presents a potential obstacle to miRNA dissemination into the peripheral circulation.

To address this issue, we reviewed a cohort of matched serum and tissue samples from pediatric medulloblastoma, ependymoma, and juvenile pilocytic astrocytoma (JPA) patients in order to identify areas of miRNA expression correlation and incongruity between the biological compartments.

\section{Methods \\ Human Patient Samples}

All patients who presented to the Connecticut Children's Medical Center pediatric neurosurgery department between 2014 and 2019 were prospectively reviewed for the presence or absence of pediatric CNS tumors. Those with radiographically diagnosed CNS tumors were eligible for enrollment in the tumor arm, and those with no evidence of a CNS tumor by radiography and clinical history were eligible for enrollment in the control arm. All oncological patients with pathology reports confirming a tumor to be a JPA, medulloblastoma, or ependymoma within 2 weeks of sample collection remained enrolled in the tumor arm.

Whole blood was obtained at presentation from all enrolled patients. In the case of patients with CNS tumors, the initial sample was acquired prior to any therapeutic interventions for the tumor. If the patient had uncontrolled hydrocephalus at presentation, it had to be treated at least 24 hours prior to sample collection. Patients with active infections were excluded. These measures were employed to avoid contaminating the miRNA expression profile with a host stress response.

Tissue samples were obtained from formalin blocks containing tumor biopsy samples after all clinically necessary tests had concluded, as determined by the lead neuropathologist. All samples were collected with patients' or their parents' consent and in accordance with institutional ethics guidelines, with the protocol approved by the Connecticut Children's Medical Center institutional review board.

Serum samples from 5 patients were included in the control arm. Patient age ranged from 1 year to 17 years at the time of enrollment/sample collection (mean 9.2 \pm 7.0 years), with a male/female ratio of 4:1. Two patients had congenital hydrocephalus, 2 had posthemorrhagic hydrocephalus, and 1 had spina bifida.

Samples from 10 patients were included in the tumor arm. Patient age ranged from 6 months to 19 years at the time of enrollment/sample collection (mean $6.5 \pm 5.4$ years), with a male/female ratio of 4:1. JPA was diagnosed in 3 patients, group 3 medulloblastoma was diagnosed in 4 patients, and anaplastic ependymoma (posterior fossa) was diagnosed in 3 patients.

\section{Serum Separation and Storage}

Blood samples were collected via peripheral venipuncture at the time of routine laboratory tests that coincided with their standard medical care. Four milliliters of blood was obtained in EDTA tubes (BD368661, Thermo Fisher Scientific) and transported at $4^{\circ} \mathrm{C}$ to the laboratory within 4 hours after venipuncture. The blood sample was centrifuged for 10 minutes at $1500 \mathrm{~g}$. The serum in the supernatant was collected into RNase-free tubes and centrifuged again for 10 minutes at $12,000 \mathrm{~g}$ at $4^{\circ} \mathrm{C}$. The supernatant was then removed and stored at $-80^{\circ} \mathrm{C}$.

\section{Serum miRNA Extraction}

Two hundred microliters of frozen-stored serum was thawed at $4^{\circ} \mathrm{C}$, mixed with $1000 \mu \mathrm{l}$ of QIAzol Lysis Reagent (Qiagen), and vortex-mixed at room temperature. After incubation for 5 minutes at room temperature, $3.5 \mu \mathrm{l}$ of synthetic Caenorhabditis elegans miRNA (cel-miR-39, $1.6 \times 10^{8}$ copies/ $\mu$ l) (Qiagen) was added and mixed with the sample. Extraction then proceeded per the miRNeasy serum/plasma manufacturer's protocol (Qiagen). All samples were eluted in $14 \mu \mathrm{l}$ of RNase-free water. The concentrations of the final RNA yield were measured using a NanoDrop 1000 spectrophotometer (Thermo Fisher Scientific).

\section{Paraffin Tissue Block miRNA Extraction}

Paraffin-embedded patient tumor tissue blocks were processed by sectioning two or three 5- to $10-\mu \mathrm{m}$-thick sections from the block surface with a sterile scalpel blade, being sure to include the tissue of interest as close to the center of the section as possible. Samples were deparaffinized and miRNA was extracted using the miRNeasy FFPE kit (Qiagen), per the manufacturer's protocol.

\section{Normal Cerebellar Tissue RNA}

Normal human cerebellar tissue RNA was commercially acquired from Thermo Fisher (\#AM7962). The patient characteristics of the RNA samples are listed in Supplemental Tables 1-8.

\section{Real-Time Polymerase Chain Reaction}

miRNA templates were maintained at $-80^{\circ} \mathrm{C}$ and thawed at $4^{\circ} \mathrm{C}$. miRNA profiling was performed using a human miRNA polymerase chain reaction (PCR) 96-well format array (MIHS-108Z; Qiagen). The array includes 84 miRNAs involved in CNS tumor biology. A set of controls was included on each plate for data analysis using the $\Delta \Delta \mathrm{Ct}$ method of relative quantification, assessment of reverse transcription performance, and assessment of PCR performance. The miScript miRNA SYBR Green-based PCR was performed, per the manufacturer's protocol, in a Bio-Rad CFX real-time (RT) PCR system (Bio-Rad), as follows: $95^{\circ} \mathrm{C}$ for 15 minutes; 40 cycles of $94^{\circ} \mathrm{C}$ for $15 \mathrm{sec}$ - 
onds; $55^{\circ} \mathrm{C}$ for 30 seconds; and $70^{\circ} \mathrm{C}$ for 30 seconds. The relative expression of individual miRNAs was calculated using the $\Delta \Delta \mathrm{Ct}$ method $(\Delta \Delta \mathrm{Ct}=\Delta \mathrm{Ct}$ test sample $-\Delta \mathrm{Ct}$ calibrator sample).

\section{Statistical Analysis}

RT-PCR reactions were analyzed and statistical computations were performed using the Qiagen Data Analysis Center (www.qiagen.com/us/shop/genes-and-pathways/ data-analysis-center-overview-page). Cel-mir-9-3p was used to assess reverse transcriptase efficiency. SNORD61 was utilized for normalization across $\mathrm{Ct}$ values. Significance for average $\Delta \mathrm{Ct}(\Delta \Delta \mathrm{Ct})$ was calculated using the Mann-Whitney U-test. Receiver operating characteristic (ROC) curves were generated using IBM SPSS software (version 26, IBM Corp.).

Significant, correlated miRNAs within serum and tumor tissue compartments were determined using the following criteria: 1) significant $(\mathrm{p}<0.05)$ concordant overexpression in both serum and tumor tissue samples, and 2) significant positive correlation in relative expression levels across matched samples. Additionally, solid tumor nodule volumes were correlated to miR expression levels as an adjunct for degree of tumor tissue-host tissue interface and distortion across brain tumor types.

The tumor nodule was defined as the homogeneously or heterogeneously enhancing region of the tumor on T1weighted gadolinium-enhanced MRI of the brain. The tumor cyst was defined as the T1 hypointense, nonenhancing region of the tumor on T1-weighted gadolinium-enhanced MRI of the brain. Volumes were calculated using the Brainlab volumetric report function after region of interest extraction facilitated by the SmartBrush application (Brainlab). All measurements were based on preoperative imaging within 1 month of surgery and simultaneous sample collection.

\section{Results}

\section{Dominant miRNA Expression Alterations}

Evaluation of miRNA alterations in the anaplastic ependymoma tissue cohort revealed 29 of 84 tested miRNAs to be significantly different from normal cerebellar tissue miRNAs. The greatest increases in expression were noted in miR-23a-3p, miR-26a-5p, and miR-9-5p.

The ependymoma cohort-matched serum miRNA profiles identified 4 of 84 tested miRNAs as having significant alterations compared with control serum samples. The greatest increase in expression was noted in miR-93a-5p. This miRNA was also the only significantly altered miRNA in the sera of ependymoma patients that was also elevated in the matched tumor tissue cohort. miR-93-5p was an average 74.1-fold higher in ependymoma tumor tissues than in control cerebellar tissues $(\mathrm{p}<0.0001)$, while this same miRNA was on average 879.6-fold higher in the serum of anaplastic ependymoma patients than in controls $(\mathrm{p}=0.046)$.

Significant medulloblastoma tissue miRNA alterations were identified in 29 of the 84 tested miRNAs. The most highly overexpressed miRNAs in the medulloblastoma tissues examined here were miR-9-5p and miR-21-5p. Only miR-15a-5p (Fold: 18.0, 95\% CI [2.8-1346.6], $\mathrm{p}=0.049$ ) was significantly altered relative to controls in the matched medulloblastoma patient serum cohort.

JPA tumor tissue samples showed significantly overexpressed levels of 46 of 84 tested miRNAs. Serum samples from the same patients demonstrated elevation in 4 of 84 tested miRNAs. miR-222 and miRs $29 \mathrm{~b}$ and $29 \mathrm{c}$ were all significantly overexpressed in the sera and tissues of patients with JPA. miR-21 was also highly overexpressed in matched sera and tissue samples, but it did not meet significance.

\section{Predictive Potential of Circulating miRNA}

Among the detected miRNA within patient sera, miR-222-3p and miR-26a-5p demonstrated significant predictive power for the presence of an intracranial JPA. miR-222-3p levels $\geq 21.9$ had a sensitivity of $88.9 \%$ and specificity of $72 \%$ (area under the curve [AUC] 0.796, p = 0.009 ), while miR-26a-5p levels $\geq 387$ had a sensitivity of $100 \%$ and specificity of $64 \%$ (AUC $0.751, p=0.027$ ) for the presence of a JPA tumor (Fig. 1). Analysis of serum miRNA in medulloblastoma patients yielded no significantly predictive miRNAs for the presence of a medulloblastoma tumor.

In the case of ependymoma patients, miR-137, miR-1385p, miR-183-5p, and miR-93-5p were significantly elevated in the sera. miR-138-5p and miR-93-5p were particularly predictive of the presence of a posterior fossa anaplastic ependymoma tumor. miR-138-5p levels $\geq 16.06$ had a sensitivity of $80 \%$ and a specificity of $67 \%$ (AUC $0.817, p=$ 0.004 ) for tumor presence, and miR-93-5p expression $\geq$ 154.48 had a sensitivity of $80 \%$ and specificity of $100 \%$ (AUC 0.821, p = 0.004) for tumor (Fig. 2).

\section{Correlation Between Sera and Tumor Tissue miRNA Expression Levels}

Four of 84 miRNAs tested in medulloblastoma patients significantly positively correlated in expression levels in the serum and tumor tissue itself (Table 1). Fourteen of 84 miRNAs tested in anaplastic ependymoma patients significantly positively correlated in expression levels between the serum and tumor tissues (Table 2). miR-93-5p, which was significantly overexpressed in the serum and tumor tissues of ependymoma patients, was also highly correlated, but its expression levels moved inversely between the two sources $\left(\mathrm{R}^{2}=-0.972, \mathrm{p}=0.026\right)$. miRs 137,138 , and 183 , which were also significantly overexpressed in the sera of anaplastic ependymoma patients, did not significantly correlate with their counterpart miRNA in matched tumor tissues.

Fifteen of 84 miRNAs tested in JPA patients were found to significantly positively correlate between serum and tissues (Table 3). miR-26a, which was predictive for a JPA tumor within this population, was also highly correlated between patient sera and patient tumor tissue. miR-222-3p did not significantly correlate with JPA tissue expression levels.

\section{Cluster Analysis}

Cluster analysis across all 84 miRNAs examined demonstrated a qualitative grouping of tissue types around immunohistochemically predefined groupings, with complete 


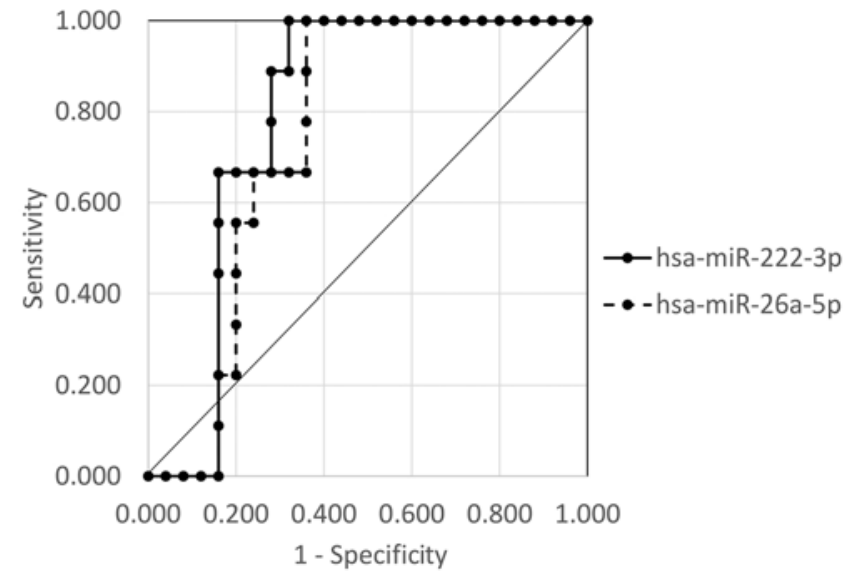

FIG. 1. ROC curve demonstrating the relative sensitivities and specificities for the presence of a JPA in measured patient sera samples versus the entire pediatric serum cohort. hsa $=$ Homo sapiens.

agreement between control and JPA tissue sample miRNA and predefined diagnosis. Ependymoma and medulloblastoma tissue demonstrated incomplete clustering (Fig. 3).

In contrast to miRNA clustering analysis with tissue samples, serum miRNA profiles produced no qualitative organization along predefined diagnostic groupings (Fig. 4).

\section{Correlation of Tumor Type Critical Serum miRNA and Tumor Volume}

JPA serum miRNA concentrations of miR-144-3p were significantly correlated $\left(R^{2}=0.865, p=0.006\right)$ with solid tumor nodule volume, as were miR-222-3p $\left(\mathrm{R}^{2}=0.735\right.$, $\mathrm{p}$ $=0.038), \operatorname{miR}-29 \mathrm{a}\left(\mathrm{R}^{2}=0.801, \mathrm{p}=0.017\right)$, and $\mathrm{miR}-29 \mathrm{c}\left(\mathrm{R}^{2}\right.$ $=0.824, \mathrm{p}=0.012$ ).

Medulloblastoma serum miRNA concentrations of miR-15a-5p $\left(\mathrm{R}^{2}=0.882, \mathrm{p}=0.002\right)$ were highly correlated with solid tumor nodule volume, as was miR-144-3p $\left(\mathrm{R}^{2}=\right.$ $0.966, \mathrm{p}<0.001)$.

There was no correlation between significantly altered serum miRNA in ependymoma patients and ependymoma tumor volume.

\section{miRNA Pathway Interactions}

TarBase analysis of the significantly differentially expressed miRNA found in our malignant tumor samples identified significant ( $p<0.0001,>12$ miRNAs) interactions with lysine degradation, proteoglycan regulation, Hippo signaling, p53 signaling, cell cycle regulation, adherens junction regulation, transforming growth factorbeta signaling, protein processing in the endoplasmic reticulum, FoxO signaling, and fatty acid biosynthesis pathways for the ependymoma cohort and lysine degradation, proteoglycan regulation, p53 signaling, and Hippo signaling in the medulloblastoma cohort. In the case of the ependymoma miRNA networks, miR-93-5p was predicted to exert significant interactions in all pathways listed above.

\section{Discussion}

This survey of matched miRNA expression patterns in the serum and tumor tissues of pediatric brain tumor patients looked to establish whether there is evidence of synchronous levels of miRNA within the two compart-

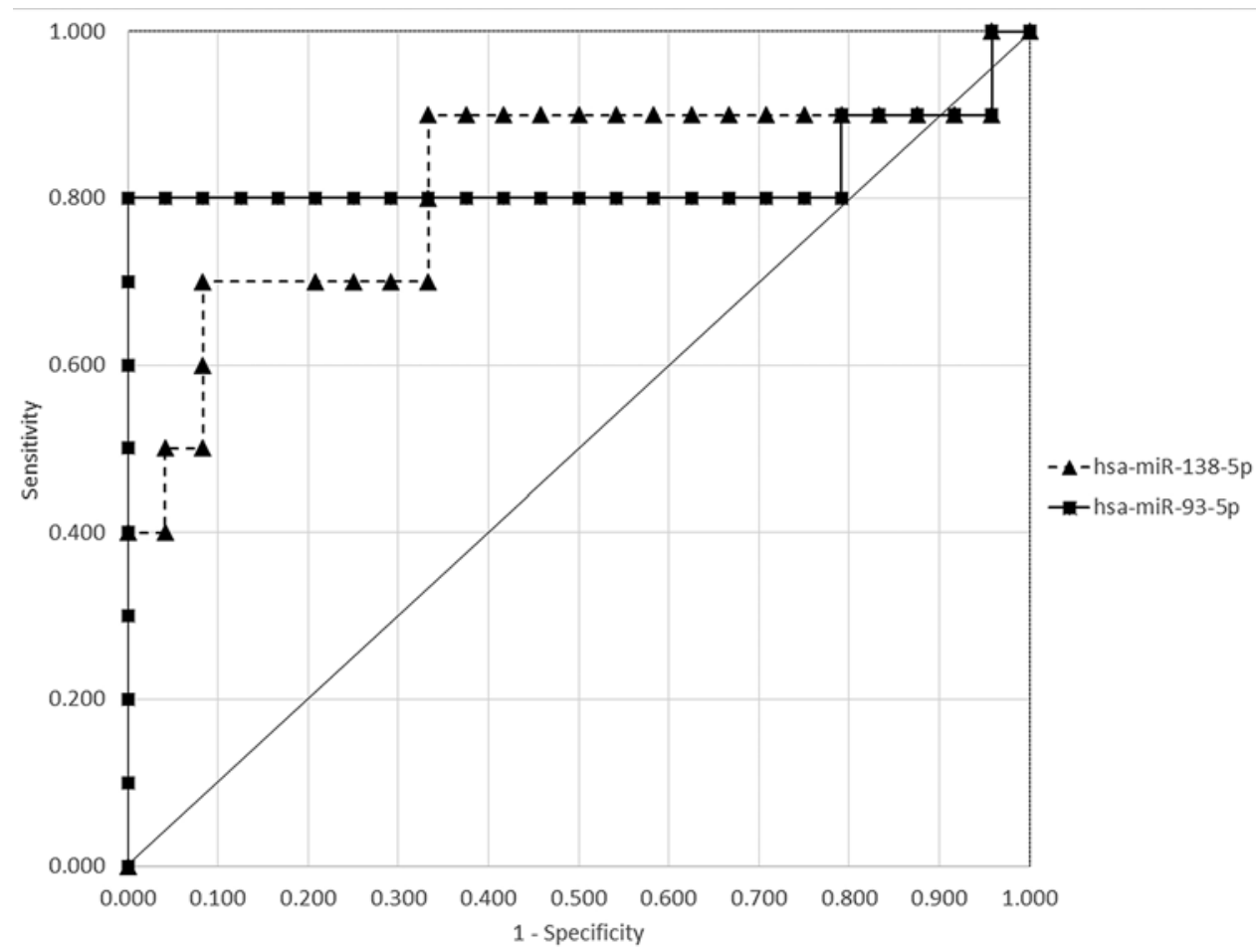

FIG. 2. ROC plot for miR-93-5p and miR-138-5p expression levels relative to the presence or absence of a posterior fossa ependymoma. 
TABLE 1. Correlation statistics of 4 miRNAs in medulloblastoma patients with matched sample significant correlations in expression levels

\begin{tabular}{lcc}
\hline \multicolumn{1}{c}{ miRNA } & Pearson Correlation Coefficient & p Value \\
\hline hsa-miR-210-3p & 0.964 & 0.018 \\
\hline hsa-miR-216a & 0.982 & 0.009 \\
\hline hsa-miR-218-5p & 0.958 & 0.021 \\
\hline hsa-miR-451a & 0.904 & 0.048 \\
\hline
\end{tabular}

hsa $=$ Homo sapiens .

ments. This is a critical knowledge gap for researchers and clinicians seeking to utilize miRNA in peripheral biofluids (i.e., urine, blood, saliva) as biomarkers for oncological processes occurring behind the blood-brain barrier. Understanding the most likely source of serum miRNA alterations in pediatric brain tumor patients is vital to provide context for these data and accurately informing its interpretation.

In this survey of neurooncological miRNA in pediatric patients with one of the 3 most common pediatric brain tumor types, only miR-26a-5p was found to be significantly overexpressed in both JPA tumor tissues and matched patient serum samples in a manner such that levels significantly correlated between the two compartments-serum and tumor tissue.

miR-26a-5p is a well-documented oncomir in gliomas, exerting negative regulatory pressure on PTEN and prohibitin, leading to increased cellular proliferation and angiogenesis in gliomas. ${ }^{14,15}$ It also plays a role in regulating neurogenesis through BDNF, ${ }^{16}$ a fact that may need to be considered when interpreting circulating miR-26a-5p levels in pediatric patients. BDNF and its regulatory molecules are dynamically expressed within different regions of the brain within the first few years of life; ${ }^{17} \mathrm{so}$, endogenous expression of miR-26a-5p may also be variable in young children. Relative levels of miR-26a-5p overexpression have also been shown to correlate with glioma WHO grade ${ }^{15}$ and ParvizHamidi et al. found elevated serum miR-26a levels to be a significant predictor of glioblastoma in patients and a more specific biomarker than the more commonly referenced miR-2 $1 .{ }^{18}$ miR-26a aside, the lack of congruity between expression levels for nearly all of the 84 miRNAs tested in all 3 pediatric brain tumor populations studied does not exclude the possibility that tumor-generated miRNAs comprise a portion of the miRNA aberrations noted in some pediatric tumor patient populations, but it strongly suggests that the preponderance of the miRNA changes observed in the serum of pediatric brain tumor patients arises from nononcologic cell types.

To further assess the hypothesis that significantly overexpressed miRNA in the serum of brain tumor patients may be related to generic inflammation in host tissues in the presence of a brain tumor, we measured tumor volumes and examined their relationship to peripheral miRNA expression levels. Larger tumors, particularly those with significant tumor-brain tissue interfaces, like JPA and medulloblastoma, would be expected to induce more robust host tissue changes through brain tissue compres-
TABLE 2. Correlation statistics of 14 miRNAs in ependymoma patients with matched sample significant correlations in expression levels

\begin{tabular}{lcr}
\hline \multicolumn{1}{c}{ miRNA } & Pearson Correlation Coefficient & $p$ Value \\
\hline hsa-miR-10b-5p & 0.968 & 0.031 \\
\hline hsa-miR-23b-3p & 0.999 & $<0.001$ \\
\hline hsa-miR-101-3p & 0.948 & 0.049 \\
\hline hsa-miR-107 & 0.983 & 0.016 \\
\hline hsa-miR-127-5p & 0.965 & 0.033 \\
\hline hsa-miR-129-5p & 0.996 & 0.004 \\
\hline hsa-miR-153-3p & 0.986 & 0.014 \\
\hline hsa-miR-184 & 0.999 & $<0.001$ \\
\hline hsa-miR-210-3p & 0.999 & $<0.001$ \\
\hline hsa-miR-217 & 0.999 & $<0.001$ \\
\hline hsa-miR-296-5p & 0.999 & 0.001 \\
\hline hsa-miR-326 & 0.999 & $<0.001$ \\
\hline hsa-miR-331-5p & 0.999 & $<0.001$ \\
\hline hsa-miR-96-5p & 0.999 & $<0.001$ \\
\hline hsa-miR-101-3p & 0.948 & 0.049 \\
\hline
\end{tabular}

sion, toxic metabolites, or invasive tumor cells. The strong correlation between miR-144 and tumor volume within both JPA and medulloblastoma cohorts and a lack of correlation between this serum miR and tumor tissue miR144 levels is in line with this hypothesis. Based on these data, serum miRNA changes are more likely to represent a host reaction to brain tumor growth than intrinsic brain tumor miRNA alterations.

The generic association between miR-144-3p and tumor dissemination or growth has been theorized in the oncology literature previously. miR-144-3p has been a frequently implicated miRNA in cancer, with downregulation of miR-144-3p being seen in a wide variety of CNS

TABLE 3. Correlation statistics of 15 miRNAs in JPA patients with matched sample significant correlations in expression levels

\begin{tabular}{lcr}
\hline \multicolumn{1}{c}{ miRNA } & Pearson Correlation Coefficient & $p$ Value \\
\hline hsa-let-7b-5p & 0.959 & 0.039 \\
\hline hsa-miR-15-5p & 0.999 & 0.001 \\
\hline hsa-miR-26a-5p & 0.951 & 0.046 \\
\hline hsa-miR-29b-3p & 0.996 & 0.004 \\
\hline hsa-miR-29c-3p & 0.954 & 0.043 \\
\hline hsa-miR-31-5p & 0.998 & 0.002 \\
\hline hsa-miR-127-5p & 0.979 & 0.020 \\
\hline hsa-miR-132-3p & 0.967 & 0.032 \\
\hline hsa-miR-149-5p & 0.966 & 0.033 \\
\hline hsa-miR-181b-5p & 0.999 & $<0.001$ \\
\hline hsa-miR-184 & 0.959 & 0.038 \\
\hline hsa-miR-200a-3p & 0.979 & 0.020 \\
\hline hsa-miR-323-5p & 0.999 & $<0.001$ \\
\hline hsa-miR-324-5p & 0.992 & 0.008 \\
\hline hsa-miR-425-5p & 0.995 & 0.005 \\
\hline
\end{tabular}




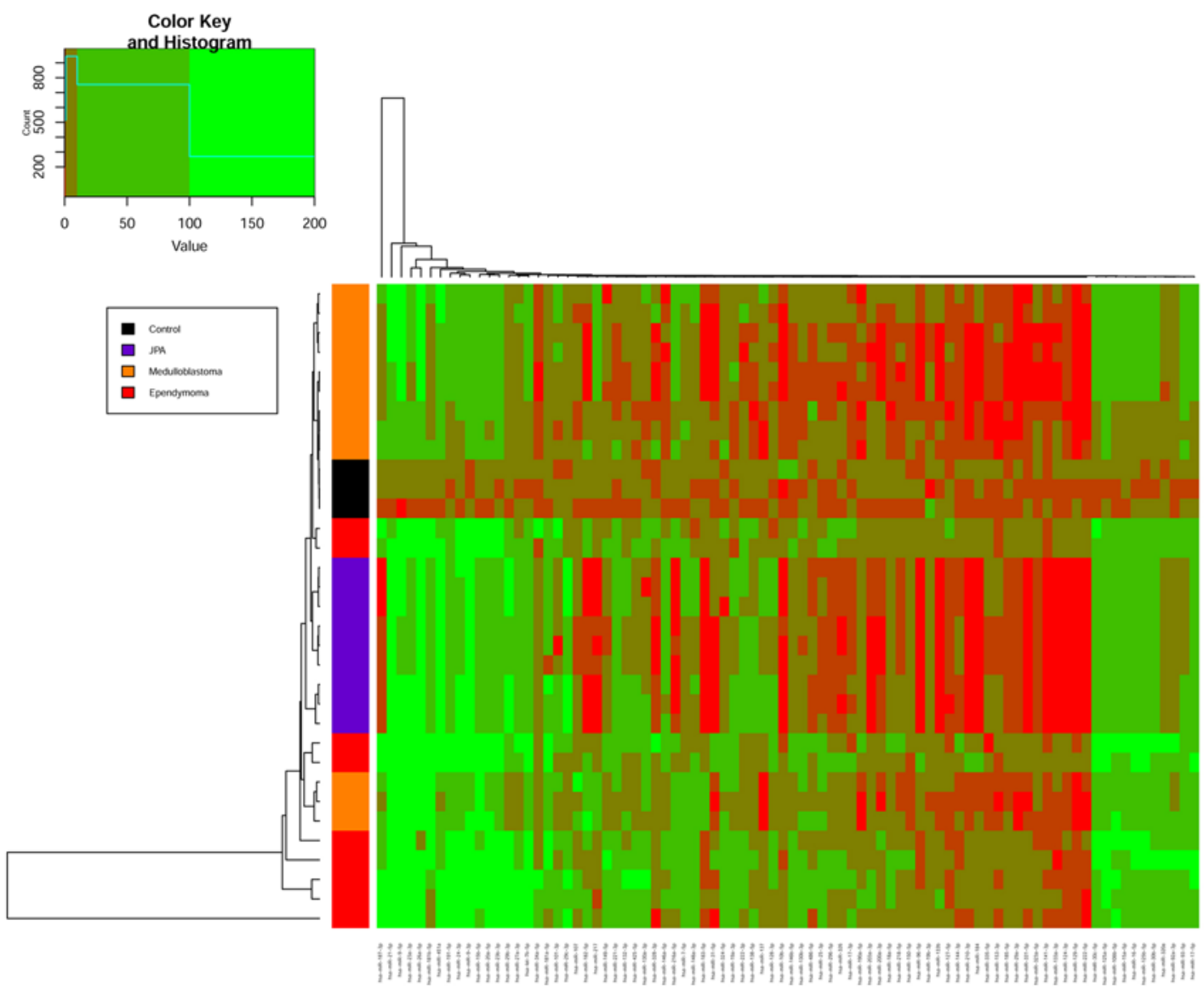

FIG. 3. miRNA heat map with average clustering analysis of tissue samples. Figure is available in color online only.

and extra-CNS tumors. ${ }^{19}$ Decreased levels of miR-144-3p in gastric, lung, head and neck, and glioma tumor tissues have been associated with increased cancer cell invasion and growth. ${ }^{20-23}$ miR-144-3p serum levels have been proposed as a diagnostic marker for colorectal cancer, esophageal cancer, hepatocellular cancer, and myocardial infarction, but this study is the first to note a relationship between brain tumor volume and serum levels of miR144-3p. ${ }^{24-27}$ Given miR-144-3p's documented tumor suppressor activity through inhibition of cell migration and its prevalence in the serum of patients with a broad range of tumor types, it is possible that elevated levels of serum miR-144-3p represent a generic host response to migratory or invasive tumor cells.

The disconnect in serum miRNA levels compared with tumor tissue miRNA levels makes serum miRNA a likely unsuitable pathway to deducing pediatric brain tumor molecular subtyping. However, these data do not exclude the possibility that serum or other peripheral, circulating miRNAs containing biofluids may be useful sources for developing diagnostic or prognostic biomarkers for pediatric brain tumors, rather it indicates that the interpretation of any miRNA aberrations detected in patient sera should be tempered by the knowledge that it likely represents reactive changes in the patient miRNome rather than that of the tumor.

CSF may be a more appropriate biofluid for mining brain tumor-specific miRNA, based on this data and the work of other authors examining serum miRNA changes in the setting of extra-CNS tumors. Armstrong et al. performed a study comparable to this one in which they examined miRNA expression levels in the urine and serum of bladder cancer patients. ${ }^{28}$ As with the current study, the authors found no significant correlation between serum miRNA and tumor tissue miRNA. Urine exosome miRNA, however, was significantly correlated with bladder cancer tissue miRNA, suggesting that tumor-derived miRNA may be better detected in relatively acellular biofluids that lie in intimate contact with the tumor, without an intervening endothelial barrier.

In the case of primary brain tumors, CSF often partially bathes tumor tissues and may collect tumor exosomes, apoptotic bodies, and naked miRNA shed from the tumors themselves. Several authors have documented elevated levels of miRNA, particularly miR-21, in the CSF exosomes of glioma and medulloblastoma patients. These miRNA signatures have been shown to be highly specific and sensitive for these select tumors in adult cohorts, but their cell-type of origin has not been verified ${ }^{29-31}$ Further studies focusing on the relative miRNA expression levels of matched CSF-derived and tumor tissue-derived samples would be important in discerning whether miRNA changes in the CSF represent commensurate changes within brain tumors themselves. If a true correlation ex- 


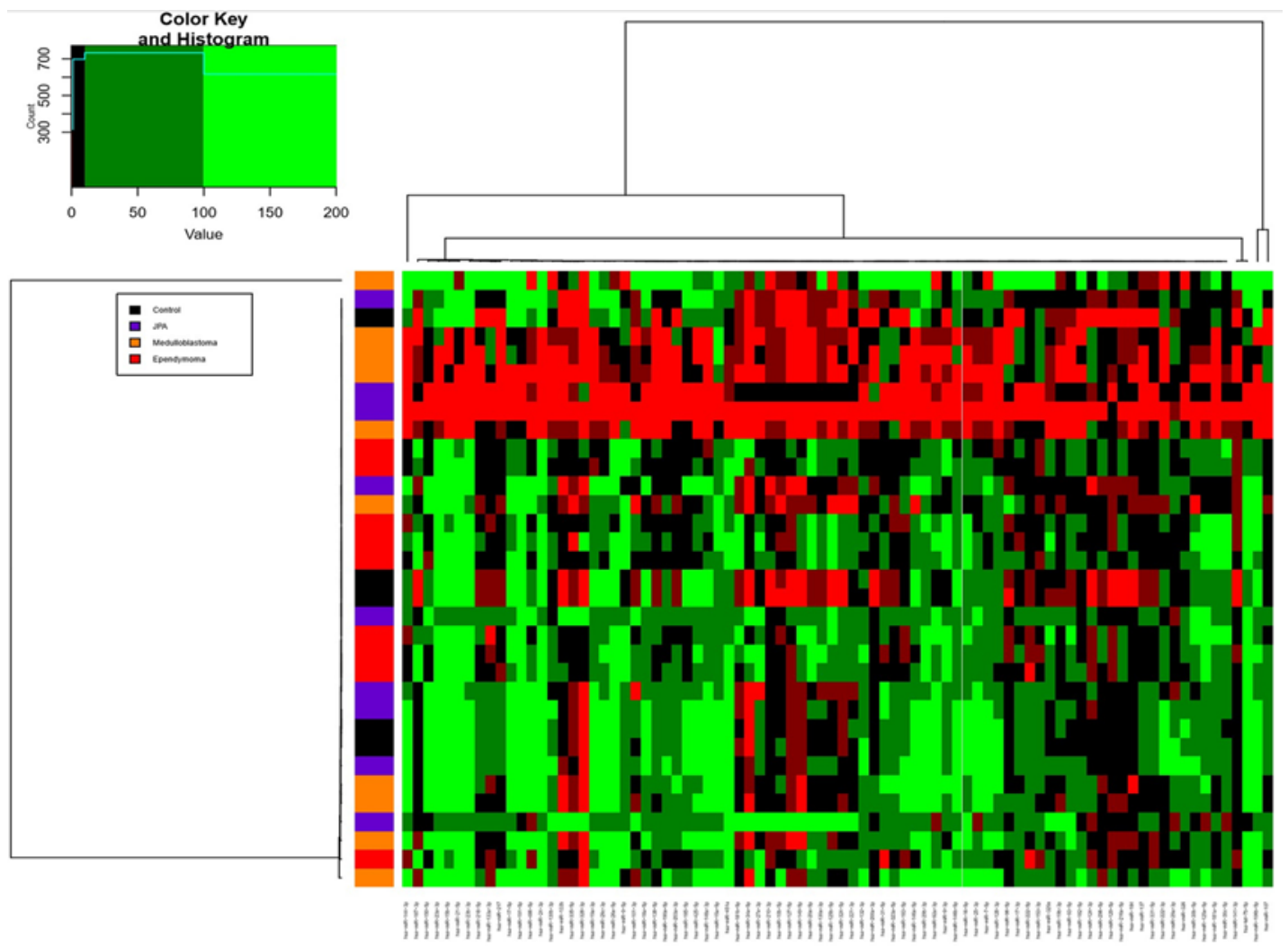

FIG. 4. miRNA heat map for serum samples across control, JPA, medulloblastoma, and ependymoma patient samples. Figure is available in color online only.

ists, then CSF miRNA profiling might be recommended as a "liquid biopsy" technique capable of extrapolating molecular changes within a tumor that could direct therapy without the need for serial tumor biopsy surgeries.

As a secondary outcome, our findings support past work noting that select serum miRNAs, regardless of their biocompartment source, do appear to strongly predict the presence and type of tumor in pediatric brain tumor patients. ${ }^{1,32-36}$ Both JPA and ependymoma patient serum miRNA profiles in this cohort displayed unique patterns of miRNA overexpression compared with controls and other tumor types. miR-26a-5p and miR-222-3p displayed high levels of sensitivity and specificity for JPA tumors within this small cohort of patients, and miRs 93-5p and 138-5p were equally predictive of an ependymoma tumor within the same cohort.

It is worth noting, however, that hierarchical cluster analysis of our patient cohort as a whole did not identify distinct groupings of serum miRNA expression levels that could be grossly related to the patient's underlying brain tumor status. Such analyses have the tendency to drown out the effects of 1 or 2 modestly differentiated variables, which supports the notion that most serum miRNA changes in brain tumor patients are not correlated to miRNA within tumors themselves. In contrast, changes in tumor tissue expression levels across the 84 miRNAs examined in this study accurately grouped 2 of 3 of the ependymoma cases, 3 of 4 of the medulloblastomas, and 3 of 3 of the JPA cases.

As serum miRNA profiles in ependymoma patients have not previously been studied, to our knowledge, the findings from this study are particularly interesting, as we demonstrated that miR-93-5p was significantly elevated in matched serum and tumor tissue samples within all 3 patients. Furthermore, it possessed an impressive $80 \%$ sensitivity and $100 \%$ specificity for ependymoma within the pooled patient population. miR-93-5p is located on 7q22.1, an oft-duplicated portion of the ependymoma genome, and it targets several genes that have been previously shown to be aberrantly expressed in group A ependymomas, including IGF2BP1 and FUS. ${ }^{37-39}$ Additionally, miR-93-5p exerts a strong regulatory function over proliferative pathways such as PI3K/AKT/TGF $\beta$, migratory pathways such as EMT (epithelial-mesenchymal transition), and over the proapoptotic gene PDCD4. The results of these interactions across a variety of cancer types, including gliomas, thyroid cancer, hepatocellular carcinoma, and breast cancer, have been to promote in vitro cell survival, migration, and chemoresistance. ${ }^{40-44}$ Elevated serum levels of miR93-5p have also been associated with more aggressive disease courses in the case of breast, lung, prostate, esophageal, laryngeal, gastric, hepatocellular, and endometrial cancers. ${ }^{45}$ Further investigation of miR-93-5p expression levels in a larger ependymoma population may reveal a correlation between serum and tumor tissue compartments that escaped the power of this preliminary study and could validate this study's findings and bolster miR-93-5p as a novel diagnostic or therapeutic target.

In total, this work strongly suggests that serum miRNA 
alterations in pediatric brain tumor patients represent a more complicated interplay of tumor and host miRNA generators than the sometimes-robust sensitivity and specificity profiles of past serum miRNA biomarker research in brain tumor patients would suggest. The results of this analysis of correlations between pediatric brain tumor patient miRNAs in serum and tumor tissues recommend that neurosurgeons and neurooncologists look at peripheral miRNA profiles with caution, as they represent potentially generic host responses to oncological pathologies rather than the excesses of dysregulated miRNA-regulated cancer pathways. Given the high sensitivity, specificity, and correlation with tumor size among JPA and medulloblastoma patients in this study, serum miRNA alterations may still be useful as a noninvasive screening tool within populations of established JPA or medulloblastoma patients for the purposes of screening during or after therapy. Such an application is well supported by this and previous research examining serum miRNA in glioma and medulloblastoma patients, and it could reduce the use of sedated MRI, as well as offer a simple adjunct screening tool between scans.

\section{Study Limitations}

This is a small study population aimed primarily at assessing the correlation between serum oncologically relevant miRNA and pediatric brain tumor tissue miRNA. The individual tumor type groups were not intended to be powered to identify within group diagnostic or prognostic markers, so these findings are presented with caution. Likewise, the lack of association noted between serum miRNA and ependymoma tumor volume in this study should not be taken as proof of noncorrelation, given the small sample size.

Additionally, the observational nature of the study does not allow for a mechanistic understanding of the lack of correlation between serum miRNA and tumor miRNA. These data do not allow for an understanding of whether a portion or any of the miRNA in the sera is derived from brain tumor tissue. Nor can we identify from this data which tissues are responsible for the miRNA changes noted between individual tumor type groups.

\section{Conclusions}

This important study has shown, within a cohort of patients with the 3 most common types of pediatric brain tumors, that serum miRNA, while possibly predictive of the presence of a brain tumor, largely does not correlate with actual tumor tissue miRNA levels. The preponderance of serum miRNA circulating within pediatric brain tumor patients likely derives from host tissues. This critical information suggests that pediatric brain tumors may cause significant perturbations in the host miRNome that could be exploited for diagnostic or prognostic purposes, but serum miRNA cannot be used directly as a lens into the majority of the JPA, medulloblastoma, or ependymoma miRNA milieu.

\section{Acknowledgments}

We thank Paul Kanev, MD, Jon Martin, MD, and Petronella Stoltz, APRN, of the Connecticut Children's Division of
Neurosurgery for their participation in screening and accruing patients. We also thank Todd Jensen, MS, for his technical assistance with specimen processing, and Shefali Thaker, BS, and Mabeline Velez, BS, for their assistance in specimen collection.

Funding for this work was provided by generous gifts from the Connecticut Brain Tumor Alliance and the Somberg Family.

\section{References}

1. Bookland M, Tang-Schomer M, Gillan E, Kolmakova A. Circulating serum oncologic miRNA in pediatric juvenile pilocytic astrocytoma patients predicts mural nodule volume. Acta Neurochir (Wien). 2018;160(8):1571-1581.

2. Feng S, Qian X, Li H, Zhang X. Combinations of elevated tissue miRNA-17-92 cluster expression and serum prostatespecific antigen as potential diagnostic biomarkers for prostate cancer. Oncol Lett. 2017;14(6):6943-6949.

3. Nonaka T, Wong DTW. Liquid biopsy in head and neck cancer: promises and challenges. J Dent Res. 2018;97(6):701708.

4. Pellatt DF, Stevens JR, Wolff RK, et al. Expression profiles of miRNA subsets distinguish human colorectal carcinoma and normal colonic mucosa. Clin Transl Gastroenterol. 2016;7:e152.

5. Tang H, Liu Q, Liu X, et al. Plasma miR-185 as a predictive biomarker for prognosis of malignant glioma. J Cancer Res Ther. 2015;11(3):630-634.

6. Wang W, Li W, Ding M, et al. Identification of miRNAs as non-invasive biomarkers for early diagnosis of lung cancers. Tumour Biol. 2016;37:16287-16293.

7. Wen Y, Han J, Chen J, et al. Plasma miRNAs as early biomarkers for detecting hepatocellular carcinoma. Int J Cancer. 2015;137(7):1679-1690.

8. Wu Q, Lu Z, Li H, et al. Next-generation sequencing of microRNAs for breast cancer detection. J Biomed Biotechnol. 2011;2011:597145.

9. Chauhan R, Lahiri N. Tissue- and serum-associated biomarkers of hepatocellular carcinoma. Biomark Cancer. 2016;8(suppl 1):37-55.

10. Sun X, Yang Z, Zhang Y, et al. Prognostic implications of tissue and serum levels of microRNA-128 in human prostate cancer. Int J Clin Exp Pathol. 2015;8(7):8394-8401.

11. Schneider A, Victoria B, Lopez YN, et al. Tissue and serum microRNA profile of oral squamous cell carcinoma patients. Sci Rep. 2018;8(1):675.

12. Hu X, Martinez-Ledesma E, Zheng S, et al. Multigene signature for predicting prognosis of patients with $1 \mathrm{p} 19 \mathrm{q}$ codeletion diffuse glioma. Neuro Oncol. 2017;19(6):786-795.

13. Taylor MD, Northcott PA, Korshunov A, et al. Molecular subgroups of medulloblastoma: the current consensus. Acta Neuropathol. 2012;123(4):465-472.

14. Huse JT, Brennan C, Hambardzumyan D, et al. The PTENregulating microRNA miR-26a is amplified in high-grade glioma and facilitates gliomagenesis in vivo. Genes Dev. 2009;23(11):1327-1337.

15. Qian X, Zhao P, Li W, et al. MicroRNA-26a promotes tumor growth and angiogenesis in glioma by directly targeting prohibitin. CNS Neurosci Ther. 2013;19(10):804-812.

16. Caputo V, Sinibaldi L, Fiorentino A, et al. Brain derived neurotrophic factor (BDNF) expression is regulated by microRNAs miR-26a and miR-26b allele-specific binding. PLoS One. 2011;6(12):e28656.

17. Webster MJ, Herman MM, Kleinman JE, Shannon Weickert C. BDNF and trkB mRNA expression in the hippocampus and temporal cortex during the human lifespan. Gene Expr Patterns. 2006;6(8):941-951.

18. ParvizHamidi M, Haddad G, Ostadrahimi S, et al. Circulating miR-26a and miR-21 as biomarkers for glioblastoma multiform. Biotechnol Appl Biochem. 2019;66(2):261-265. 
19. Wang W, Peng B, Wang D, et al. Human tumor microRNA signatures derived from large-scale oligonucleotide microarray datasets. Int J Cancer. 2011;129(7):1624-1634.

20. Chen YJ, Guo YN, Shi K, et al. Down-regulation of microRNA-144-3p and its clinical value in non-small cell lung cancer: a comprehensive analysis based on microarray, miRNAsequencing, and quantitative real-time PCR data. Respir Res. 2019;20(1):48

21. Lin L, Zheng Y, Tu Y, et al. MicroRNA-144 suppresses tumorigenesis and tumor progression of astrocytoma by targeting EZH2. Hum Pathol. 2015;46(7):971-980.

22. Mushtaq F, Zhang J, Li J. miR-144 suppresses cell proliferation and invasion in gastric cancer through downregulation of activating enhancer-binding protein 4. Oncol Lett. 2019;17(6):5686-5692.

23. Zhang SY, Lu ZM, Lin YF, et al. miR-144-3p, a tumor suppressive microRNA targeting ETS-1 in laryngeal squamous cell carcinoma. Oncotarget. 2016;7(10):11637-11650.

24. $\mathrm{Pu} \mathrm{C}$, Huang $\mathrm{H}$, Wang Z, et al. Extracellular vesicle-associated mir-21 and mir-144 are markedly elevated in serum of patients with hepatocellular carcinoma. Front Physiol. 2018;9:930.

25. Sharma P, Saraya A, Sharma R. Potential diagnostic implications of miR-144 overexpression in human oesophageal cancer. Indian J Med Res. 2016;143(suppl):S91-S103.

26. Tan Y, Lin JJ, Yang X, et al. A panel of three plasma microRNAs for colorectal cancer diagnosis. Cancer Epidemiol. 2019;60:67-76.

27. Velle-Forbord T, Eidlaug M, Debik J, et al. Circulating microRNAs as predictive biomarkers of myocardial infarction: evidence from the HUNT study. Atherosclerosis. 2019;289:1-7.

28. Armstrong DA, Green BB, Seigne JD, et al. MicroRNA molecular profiling from matched tumor and bio-fluids in bladder cancer. Mol Cancer. 2015;14:194.

29. Baraniskin A, Kuhnhenn J, Schlegel U, et al. Identification of microRNAs in the cerebrospinal fluid as biomarker for the diagnosis of glioma. Neuro Oncol. 2012;14(1):29-33.

30. Shi R, Wang PY, Li XY, et al. Exosomal levels of miRNA-21 from cerebrospinal fluids associated with poor prognosis and tumor recurrence of glioma patients. Oncotarget 2015;6(29):26971-26981.

31. Zorofchian S, Iqbal F, Rao M, et al. Circulating tumour DNA, microRNA and metabolites in cerebrospinal fluid as biomarkers for central nervous system malignancies. J Clin Pathol. 2019;72(4):271-280.

32. Ivo D'Urso P, Fernando D'Urso O, Damiano Gianfreda C, et al. miR-15b and miR-21 as circulating biomarkers for diagnosis of glioma. Curr Genomics. 2015;16(5):304-311.

33. Regazzo G, Terrenato I, Spagnuolo M, et al. A restricted signature of serum miRNAs distinguishes glioblastoma from lower grade gliomas. J Exp Clin Cancer Res. 2016;35(1):124.

34. Yang C, Wang C, Chen X, et al. Identification of seven serum microRNAs from a genome-wide serum microRNA expression profile as potential noninvasive biomarkers for malignant astrocytomas. Int J Cancer. 2013;132(1):116-127.

35. Yu X, Li Z. Serum microRNAs as potential noninvasive biomarkers for glioma. Tumour Biol. 2016;37(2):1407-1410.

36. Zhi F, Shao N, Wang R, et al. Identification of 9 serum microRNAs as potential noninvasive biomarkers of human astrocytoma. Neuro Oncol. 2015;17(3):383-391.

37. Mack SC, Pajtler KW, Chavez L, et al. Therapeutic targeting of ependymoma as informed by oncogenic enhancer profiling. Nature. 2018;553(7686):101-105.
38. National Center for Biotechnology Information. MIR93: microRNA 93 [Homo sapiens (human)]. Accessed March 11, 2020. https://www.ncbi.nlm.nih.gov/gene/407050

39. Yao Y, Mack SC, Taylor MD. Molecular genetics of ependymoma. Chin J Cancer. 2011;30(10):669-681.

40. Chen Q, Qin R, Fang Y, Li H. Berberine sensitizes human ovarian cancer cells to cisplatin through miR-93/PTEN/Akt signaling pathway. Cell Physiol Biochem. 2015;36(3):956965.

41. Ji C, Liu H, Yin Q, et al. miR-93 enhances hepatocellular carcinoma invasion and metastasis by EMT via targeting PDCD4. Biotechnol Lett. 2017:39(11):1621-1629.

42. Wang Q, Su C, Li J, Wei C. Mechanism of the enhancing effects of miR-93 on resistance of breast cancer MCF-7 cells to adriamycin. Oncol Lett. 2018;16(3):3779-3783.

43. Wu H, Liu L, Zhu JM. MiR-93-5p inhibited proliferation and metastasis of glioma cells by targeting MMP2. Eur Rev Med Pharmacol Sci. 2019;23(21):9517-9524.

44. Yang W, Bai J, Liu D, et al. MiR-93-5p up-regulation is involved in non-small cell lung cancer cells proliferation and migration and poor prognosis. Gene. 2018;647:13-20.

45. Gao Y, Deng K, Liu X, et al. Molecular mechanism and role of microRNA-93 in human cancers: a study based on bioinformatics analysis, meta-analysis, and quantitative polymerase chain reaction validation. $J$ Cell Biochem. 2019;120(4):6370-6383.

\section{Disclosures}

The authors report no conflict of interest concerning the materials or methods used in this study or the findings specified in this paper.

\section{Author Contributions}

Conception and design: Bookland, Kolmakova. Acquisition of data: Gillan, Song, Kolmakova. Analysis and interpretation of data: Bookland. Drafting the article: Bookland, Kolmakova.

Critically revising the article: Bookland. Reviewed submitted version of manuscript: all authors. Approved the final version of the manuscript on behalf of all authors: Bookland. Statistical analysis: Bookland. Administrative/technical/material support: Bookland. Study supervision: Bookland.

\section{Supplemental Information \\ Online-Only Content}

Supplemental material is available with the online version of the article.

Supplemental Tables 1-8. https://thejns.org/doi/suppl/10.3171/ 2020.2.PEDS19715.

\section{Previous Presentations}

Portions of the data presented in this manuscript were accepted as an e-poster presentation for the 2020 AANS Annual Scientific Meeting.

\section{Correspondence}

Markus Bookland: Connecticut Children's, Hartford, CT. mbookland@connecticutchildrens.org. 